\title{
Optical fiber with dual cores suspended in air
}

\author{
Zheng Gang Lian, Xian Feng, Peter Horak, Limin Xiao, Yoonchan Jeong, Nicholas White, Ken \\ Frampton, John A. Tucknott, Harvey Rutt, David N Payne, Will Stewart and Wei H Loh* \\ Optoelectronics Research Centre, University of Southampton, Southampton SO17 1BJ, United \\ Kingdom \\ *Corresponding author: whl@orc.soton.ac.uk
}

\begin{abstract}
We report the successful fabrication of a new type of dual core fiber, comprising 2 optical cores suspended in air. The cores are sub-micron in size, each held in place by 2 thin glass membranes attached to the cladding. The successful realisation of this fiber should open up a range of interesting applications in a variety of different areas.
\end{abstract}

OCIS Codes: $060.2280,060.4005$

\section{Introduction}

The last decade has seen considerable advances in optical fiber fabrication techniques and processes, resulting in the realisation of increasingly complex fiber structures, such as photonic crystal fibers [1]. Much of this effort has been aimed at tailoring the dispersion profiles, tighter confinement to enhance nonlinear optical effects, reducing transmission losses, or increasing effective mode areas to scale up to higher powers of operation. In this current work, we report on the fabrication of a new type of fiber, one where the fiber cores would be allowed to physically move and optically interact with each other; conceptually speaking, we wish to introduce optical fibers with optomechanical or MEMS-type functionalities.

The basic idea behind this dual core fiber is to have two cores inside the fiber, with each core suspended in air by two thin glass membranes, and held sufficiently close to each other such that the two cores can be coupled through their evanescent fields. Such a fiber would offer quite interesting applications in the broad field of sensing (e.g. as highly sensitive pressure, vibration, or pollution sensors). More unusually, if the cores are small enough and the membranes holding them sufficiently thin, such a structure could conceivably be deployed as a "smart" fiber, where the optical coupling between the cores automatically adjusts to the optical signal propagating through them, as a result of one core moving in direct response to forces from the optical signal in the second core [2], similar to the optical tweezers effect.

\section{Fabrication}

Lead silicate (Schott SF57) glass was used for the fabrication of this dual core fiber. The glass has a softening temperature of $519^{\circ} \mathrm{C}$ [3], enabling the glass extrusion technique [4] to be adapted to produce the desired preform structure. Fig. 1(a) shows the cross section of the stainless steel extrusion die employed to fabricate the preform. Two circular holes (cores) with diameter of $1.0 \pm 0.1 \mathrm{~mm}$ are located in the middle, with two slots (struts) of $11.5 \mathrm{~mm}$ long and $0.5 \mathrm{~mm}$ width. The two extruded struts were initially designed to be parallel, but it proved difficult to extrude the glass through such a die with the two parallel slots so close together, due to the high loads ( $\sim$ half a ton) needed to be applied to the glass during this extrusion process. Consequently, a novel curved strut preform design was adopted, with the struts expected to straighten themselves during the fiber drawing process, allowing us to fabricate the dual core fiber with the two cores very close together.

For the extrusion of the preform, the glass was heated to the desired temperature and slowly pushed through the die to form the dual-core preform; the cross-section of the preform is shown in Fig. 1(b). The extruded preform had an outer diameter of $16 \mathrm{~mm}$. The preform was then placed on the fiber draw tower and caned down to $1.5 \mathrm{~mm}$ in diameter. This was finally drawn into fiber using the cane-in-tube method, where the tube $(1.8 \mathrm{~mm}$ inner diameter / $12.0 \mathrm{~mm}$ outer diameter) was also extruded using the same SF57 glass. A gentle vacuum was applied in the gap between the cane and tube during the fiber drawing. Fig. 1(c) is a transmitted optical microscope image that shows the cross-section of the dual core fiber. The fiber has a diameter of $135 \mu \mathrm{m}$, and the two cores inside are clearly visible. 

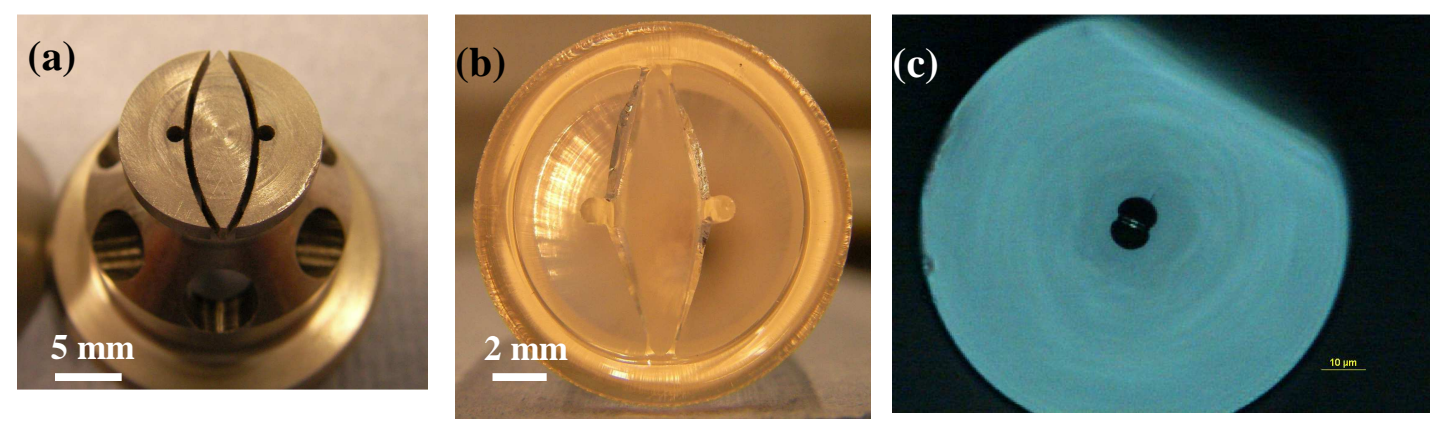

Fig. 1: (a) Top view of the extrusion die; (b) cross section of the resulting extruded glass preform and (c) transmitted optical microscope image of the cross section of the final dual core fiber.

\section{Analysis}

The features within the fiber were characterised using a Scanning Electron Microscope (SEM), shown in Fig 2. Both the two cores are suspended in air by two $11 \mu \mathrm{m}$ long, 150nm thick glass membranes. The cores became elongated in the direction of the struts due to surface tension effects during the fiber drawing process and are therefore elliptical in shape. The dimensions of the cores are $\sim 0.5 \mu \mathrm{m} \times 1.0 \mu \mathrm{m}$. The curved struts in the preform, which was shown in Fig. 1(b), had straightened themselves into parallel thin glass membranes as anticipated, bringing the two cores close together. The separation between two cores was less than $1.0 \mu \mathrm{m}$; this is necessary in order to obtain good optical interaction between the cores.

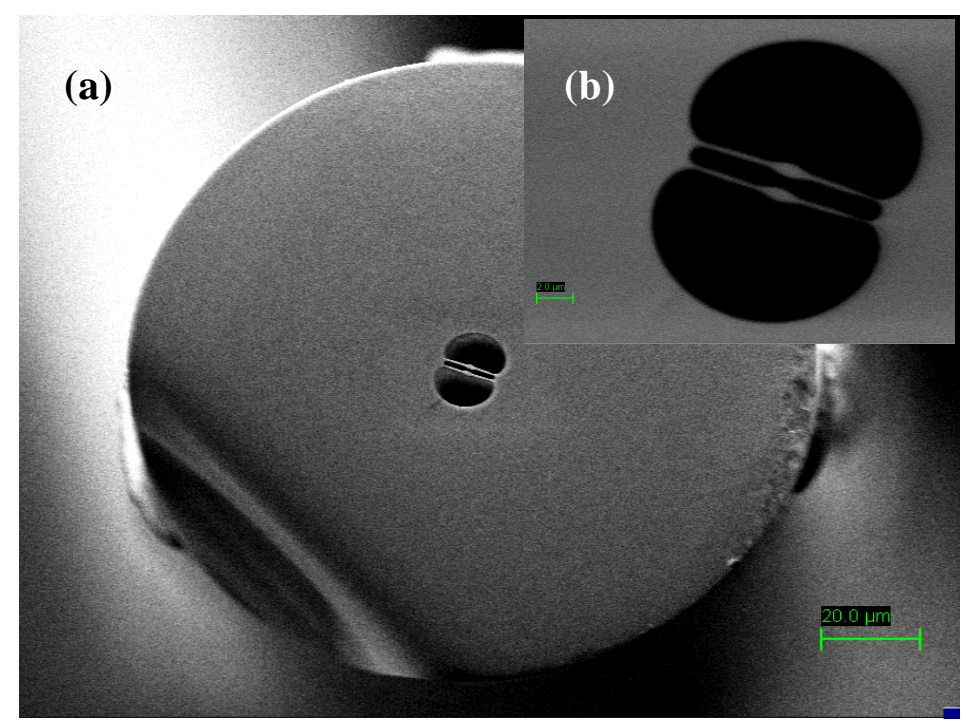

Fig. 2: The cross section of the dual core fiber, viewed using a Scanning Electron Microscope. (a) the fiber, which has an outer diameter of $135 \mu \mathrm{m}$; (b) the magnified core region. The separation between the cores is $700 \mathrm{~nm}$.

Preliminary measurements of the propagation loss of the fiber at $1550 \mathrm{~nm}$ were performed with freespace coupling from a laser diode and the fiber cut-back method with an initial fiber length of $1.3 \mathrm{~m}$. The propagation loss of the dual core fiber was $11 \mathrm{~dB} / \mathrm{m}$, as shown in Fig. 3, launching into both cores. At this stage, we speculate that much of the loss contribution is likely due to scattering at the glass-air interfaces. 


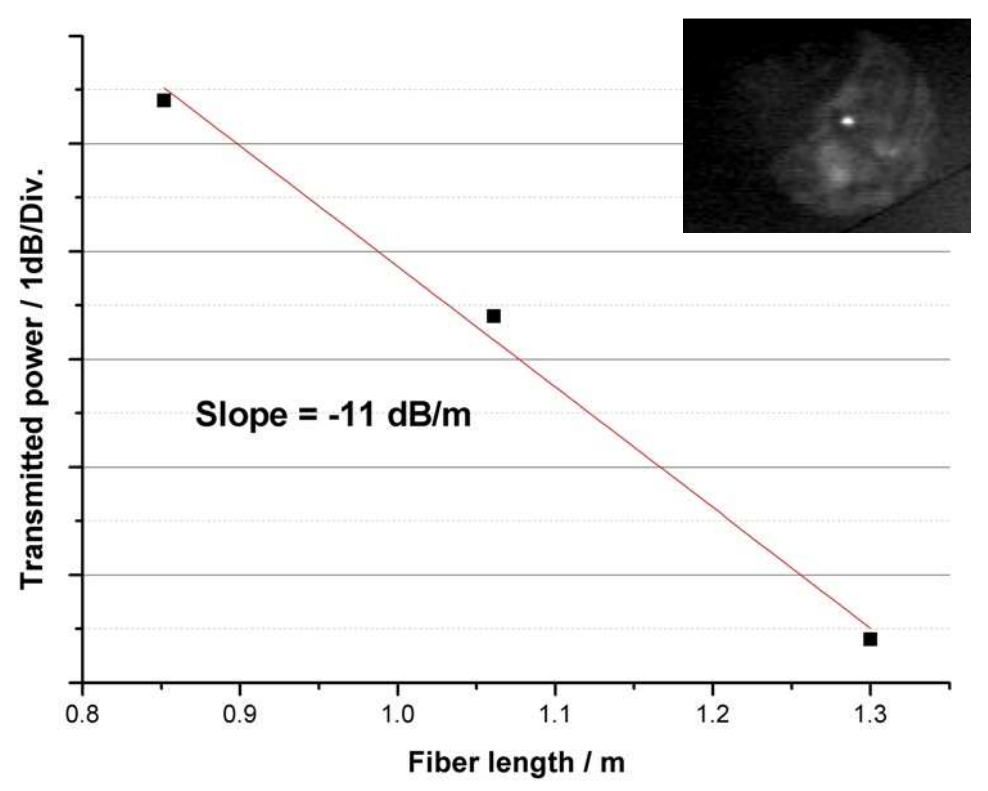

Fig. 3 Cutback loss measurement for the fiber (inset) infrared camera image of the light at $1550 \mathrm{~nm}$ transmitted by the dual core fiber.

Numerical calculations based on the feature dimensions from the SEM pictures indicate that this fiber is currently multimoded (Fig. 4), due to the ellipticity of the core and the relatively high refractive index of the lead silicate glass $(n=1.8$ at $1550 \mathrm{~nm} \mathrm{[3]).} \mathrm{Reducing} \mathrm{the} \mathrm{size} \mathrm{of} \mathrm{the} \mathrm{core} \mathrm{and/or} \mathrm{the}$ refractive index should enforce single mode behavior. At these core sizes and glass membrane dimensions, the mechanical resonances are estimated to be in the range $10 \mathrm{MHz}-100 \mathrm{MHz}$.
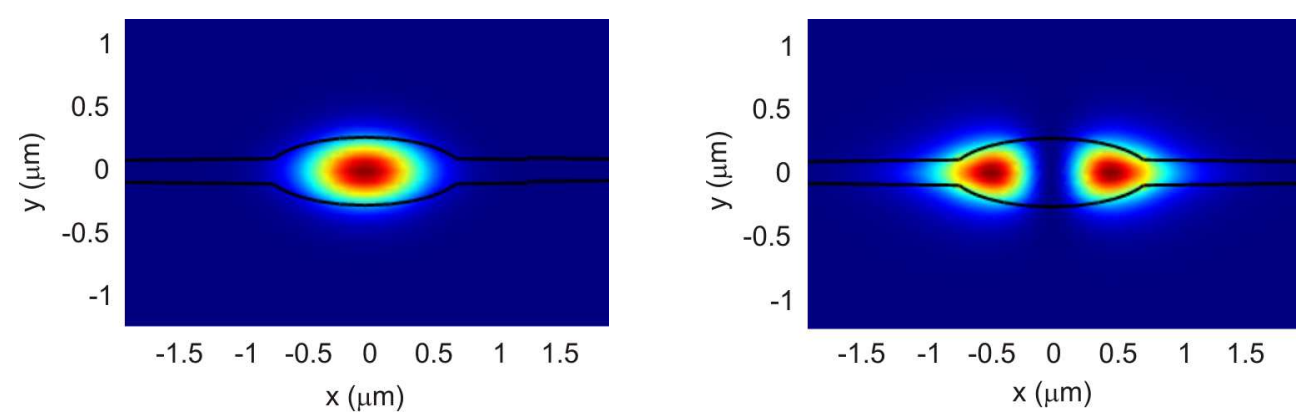

Fig. 4 Modal calculations using the dimensions of the core and glass membrane structure in Fig. 2, showing that more than one mode is supported. The modes of only one core are shown here.

\section{Conclusion}

We have fabricated a new type of optical fiber consisting of 2 sub-micron sized cores suspended in air inside the fiber, and attached to the outer glass cladding by 2 thin glass membranes. Such a fiber type should open up new possibilities in fiber functionality, such as movable MEMS-like structures inside the fiber. Potential applications include optical sensing, "smart" fibers, and optomechanically tunable fibers. We gratefully acknowledge support for this work from the Engineering and Physical Sciences Research Council (EPSRC) through funding for the EPSRC Centre for Innovative Manufacturing in Photonics, and the "Photonic Hyperhighway" Programme Grant. Y. Jeong is now with the School of Electrical Engineering, Seoul National University, Korea.

\section{References}

1. P. Russell, "Photonic crystal fibre," Science 299, 358-362 (2003).

2. M. L. Povinelli et al., "Evanescent wave bonding between optical waveguides," Opt. Lett. 30, 3042 3044 (2005).

3. Schott, Data sheet for SF57, S.G. Company, 2001.

4. X. Feng et al., "Extruded singlemode, high nonlinearity tellurite glass holey fibre," Electron. Lett. 41, 835-837 (2005). 